\title{
Impact of Corticosteroids on Mortality in Patients with Acute Respiratory Distress Syndrome: A Systematic Review and Meta-analysis
}

\author{
Nobuyuki Horita ${ }^{1,2}$, Satoru Hashimoto ${ }^{3}$, Naoki Miyazawa ${ }^{2}$, Hiroyuki Fujita ${ }^{4}$, Ryota Kojima ${ }^{2}$, \\ Miyo Inoue $^{2}$, Atsuhisa Ueda ${ }^{1}$, Yoshiaki Ishigatsubo ${ }^{1}$ and Takeshi Kaneko ${ }^{5}$
}

\begin{abstract}
Objective The impact of corticosteroids on acute respiratory distress syndrome (ARDS) mortality remains controversial following the publication of numerous trials, observational studies and meta-analyses. An updated meta-analysis is warranted, as a few original studies on this topic have been published since the last meta-analysis.

Methods We searched for eligible articles using four databases. In particular, we included full-length original articles providing sufficient data for evaluating the impact of corticosteroid treatment on adult ARDS mortality in the form of odds ratios. A fixed model with the confidence interval method was used. An assessment of publication bias and sensitivity analyses were also conducted.

Results We included 11 of 185 articles. The pooled odds ratio for corticosteroids with respect to all-cause mortality involving 949 patients was 0.77 [95\% confidence interval (CI): 0.58-1.03, $\mathrm{p}=0.079$ ] with strong heterogeneity $\left(\mathrm{I}^{2}=70 \%, \mathrm{p}<0.001\right)$. The results of the sensitivity analysis, Begg-Kendall test $(\tau=0.53, \mathrm{p}=0.024)$ and funnel plot consistently suggested the existence of strong publication bias. After six potentially unpublished cohorts were filled using Duval's trim and fill method, the pooled odds ratio shifted to $1.11(95 \% \mathrm{CI}$ 0.86-1.44, $\mathrm{p}=0.427$ ). In addition, the sensitivity analyses suggested that corticosteroid treatment has a different impact on mortality depending on the comorbidities and trigger events.

Conclusion We were unable to confirm, based on the data of published studies, the favorable impact of corticosteroid therapy on mortality in overall ARDS cases. Published articles exhibit strong publication bias, and previous meta-analyses may be affected by this publication bias. Further research focusing on pathophysiology- or trigger event-specific ARDS is anticipated.
\end{abstract}

Key words: acute lung injury, prognosis, corticosteroids, publication bias

(Intern Med 54: 1473-1479, 2015)

(DOI: 10.2169/internalmedicine.54.4015)

\section{Introduction}

Acute respiratory distress syndrome (ARDS) continues to play a major role in the mortality of critically ill patients worldwide (1). The development of ARDS is a consequence of alveolar injury related to the effects of a complex net- work of cytokines and other pro-inflammatory compounds that initiate and amplify the inflammatory response (2). It therefore appears to be a reasonable strategy to treat ARDS patients with immunosuppressants capable of potentially controlling the inflammatory response in the lungs (2). Corticosteroids have been by far the most widely used medications for ARDS since Ashbaugh reported the first cases of

\footnotetext{
${ }^{1}$ Department of Internal Medicine and Clinical Immunology, Yokohama City University Graduate School of Medicine, Japan, ${ }^{2}$ Department of Respiratory Medicine, Saiseikai Yokohamashi Nanbu Hospital, Japan, ${ }^{3}$ Department of Anesthesiology and Intensive Care, Kyoto Prefectural University of Medicine, Japan, ${ }^{4}$ Department of Hematology, Saiseikai Yokohamashi Nanbu Hospital, Japan and ${ }^{5}$ Respiratory Disease Center, Yokohama City University Medical Center, Japan

Received for publication September 1, 2014; Accepted for publication October 27, 2014

Correspondence to Dr. Nobuyuki Horita, nobuyuki_horita@yahoo.co.jp
} 
this clinical entity (2-5). Nonetheless, the impact of corticosteroids on the mortality of ARDS remains controversial following the publication of numerous trials and observational studies (6-19). Meta-analyses of these studies have indicated that the use of corticosteroids has only a marginal impact in reducing mortality in ARDS cases (20-28), although it is clear that corticosteroids improve gas exchange, decrease the duration of mechanical ventilation and decrease the length of stay in the intensive care unit $(23,25,26)$. Over the last two decades, most studies investigating ARDS have used the American-European Consensus Conference (AECC) definition (1). This widely used definition is helpful for epidemiological applications and recruiting patients for clinical trials (2). However, the heterogeneity of AECCdefined ARDS is a considerable disadvantage. Even if a particular treatment has an excellent therapeutic effect in limited subgroups, it may be harmful in other subgroups (2). As a result, it remains difficult to determine effective treatment strategies for ARDS cases overall. The Berlin definition of ARDS was proposed in 2012. Although it has slightly better predictive validity for mortality compared to the AECC definition, the Berlin definition is basically the same as the AECC definition and also contains a heterogeneous disease category (29).

A few original studies on this topic have been published since the last meta-analysis (28), and an updated metaanalysis is therefore warranted. The aim of the current study is to reevaluate the impact of corticosteroid therapy on the mortality of ARDS. We also conducted sensitivity analyses to confirm the results of the meta-analysis.

\section{Materials and Methods}

\section{Study search and evaluation}

Institutional review board approval and patient consent were not required due to the nature of this study. The current meta-analysis was conducted in compliance with the PRISMA check list (30).

Two investigators independently searched for eligible articles using the MEDLINE, EMBASE, Web of Science and Cochrane Databases as of January 2014. The following search formula was used for MEDLINE: (((“ARDS” OR "acute respiratory distress syndrome" OR "adult respiratory distress syndrome” OR “ALI" OR "acute lung injury”) AND ("steroid" OR "steroids" OR "corticosteroid" OR "corticosteroids" OR "prednisolone" OR "methylprednisolone" OR "methylprednisolone" OR "dexamethasone" OR "hydrocortisone" OR "cortisone" OR "betamethasone") AND ("hazard ratio" OR "relative risk" OR "odds ratio")) OR ((“ARDS" [title] OR "acute respiratory distress syndrome" [title] OR "adult respiratory distress syndrome" [title] OR "ALI" [title] OR "acute lung injury" [title]) AND ("steroid" [title] OR "steroids" [title] OR "corticosteroid" [title] OR "corticosteroids" [title] OR "prednisolone" [title] OR "methylprednisolone" [title] OR "methylprednisolone" [title] OR "dexamethasone" [title] OR "hydrocortisone" [title] OR "cortisone" [title] OR "betamethasone" [title]))) AND ("mortality" OR "prognosis" OR "death"). We used similar search words for other databases. Articles included in the authors' reference lists were also considered as candidates.

The eligibility criterion for the current meta-analysis was a full-length original article that provided sufficient data to evaluate the impact of corticosteroid treatment on the mortality of adult ARDS and/or acute lung injury (ALI) in the form of odds ratios (ORs). If the article did not report mortality in the form of an OR, articles that provided sufficient data to calculate the OR were included. Cases of ARDS and ALI as defined by the AECC or Berlin definition were preferred $(5,29)$, although other definitions were generally allowed if the authors of the original study stated that the patients had ARDS. Observational studies were included along with randomized controlled trials (RCTs). The existence of comorbidities and/or trigger events was not a reason for exclusion. We did not place any restrictions on the type of corticosteroids or language of publication. We excluded articles describing less than 10 cases, as we regarded these articles as being case series rather than trials or cohort studies. Articles focusing on ARDS prevention with corticosteroids and single-arm studies were also excluded. Duplicate use of the same data was carefully evaluated. The quality of eligible trials was assessed using the Chalmers checklist, in which 100 indicates the best score (31). This checklist gives large scores for the use of randomization and blinding procedures and generally assigns poor scores to observational studies. Low quality as per the Chalmers checklist was not a reason for exclusion.

\section{Data extraction}

If an original article evaluated two or more types of mortality, the primary endpoint was preferred and in-hospital mortality was preferred to intensive care unit mortality. We divided the doses of corticosteroids into high and low categories, with a cutoff value for methylprednisolone of $10 \mathrm{mg}$ / $\mathrm{kg} /$ day or $500 \mathrm{mg} /$ day equivalent for the purpose of the sensitivity analysis. Most of the articles clearly used much higher or lower doses than the cutoff values. We divided ARDS into early and late disease, with a cutoff of 14 days since onset, intubation, diagnosis or intensive care unit admission. Non-English articles were evaluated only for English abstracts, figures and tables.

\section{Statistics}

We used a fixed model with the confidence interval (CI) method (32) to estimate the pooled OR. The heterogeneity among the original studies was evaluated according to (i) the chi-square distribution test with a rejection region of $\mathrm{p}=$ 0.1 and (ii) the $\mathrm{I}^{2}$ statistics test whereby $\mathrm{I}^{2}<0 \%$ indicates no heterogeneity, $0 \%<\mathrm{I}^{2}<25 \%$ indicates mild heterogeneity, $25 \% \leq \mathrm{I}^{2}<50 \%$ indicates moderate heterogeneity, $50 \% \leq \mathrm{I}^{2}<75 \%$ indicates strong heterogeneity and $75 \% \leq \mathrm{I}^{2}$ indicates very strong heterogeneity (33). A funnel plot and Begg's rank 


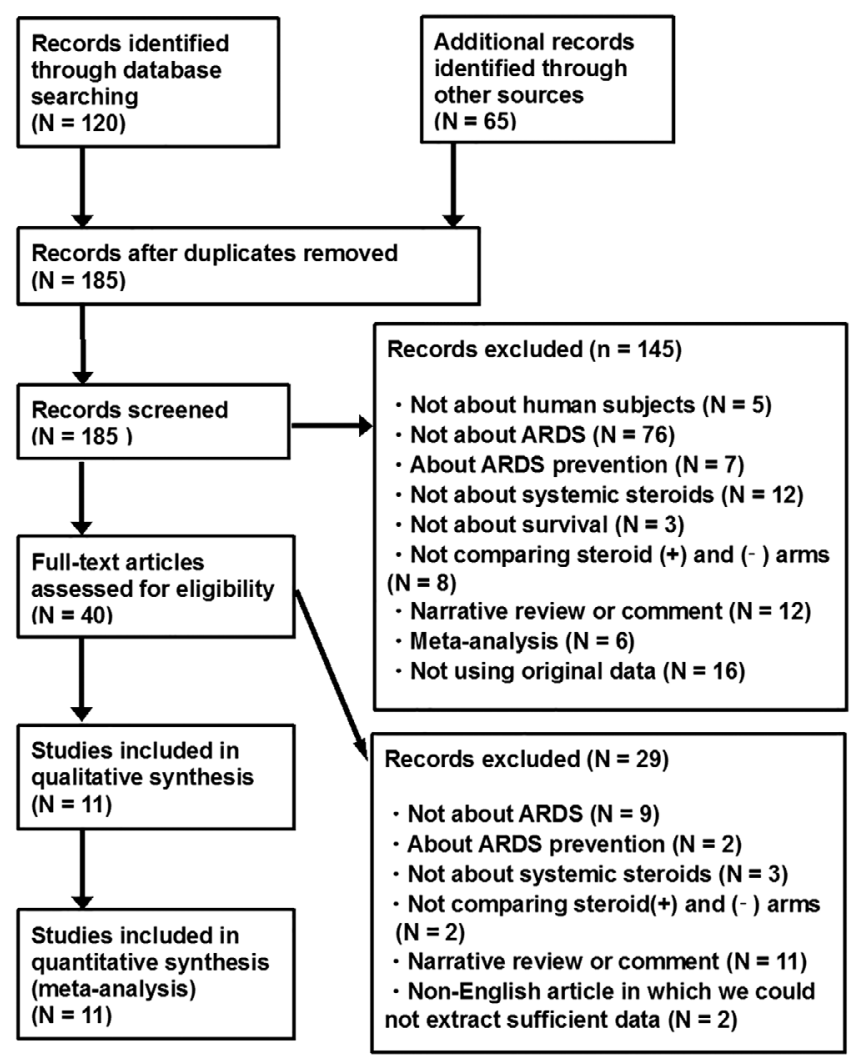

Figure 1. Flow chart of the study search. N: Number of articles

correlation test using the Kendall rank correlation test with a rejection region of $\mathrm{p}=0.1$ were used to assess the degree of publication bias (34). If publication bias was suspected, we used Duval's trim and fill method to correct for the bias $(35,36)$. Sensitivity analyses were also performed by comparing a variety of subgroups using the rejection region for the sensitivity of interaction of $\mathrm{p}=0.1$ (37). All analyses were performed using the Excel Toukei version 5.0 software program (SSRI, Tokyo, Japan).

\section{Results}

\section{Study search}

We screened 120 potentially eligible articles from database searches and 65 potentially eligible articles from authors' reference lists. Of the 185 articles, 145 were excluded based on the title and abstract and 29 were excluded after considering the full text. A total of 11 articles were finally included in the current analysis (Fig. 1). The 11 eligible articles consisted of four RCTs, one secondary analysis of an RCT and six observational studies (Table), all published between 1987 and 2012. The number of patients in each cohort ranged from 14 to 208, with a median of 48 . The total number of included patients was 949 , of whom 461 were treated with corticosteroids and 488 were treated without corticosteroids. The rates of mortality were 393/949 (41.4\%), $174 / 461(37.7 \%)$ and $219 / 488(44.9 \%)$ for all cases, corticosteroid-treated cases and non-corticosteroidtreated cases, respectively. The quality score ranged from 13 to 62 , with a median of 40 , which indicated that the quality of these studies was not always excellent (Table). In addition, the OR differed considerably, ranging from 0.01 to 2.52. Each of the three studies with the largest sample size accounted for nearly $20 \%$ of the total weight (Fig. 2).

\section{Meta-analysis of all studies}

A fixed-model meta-analysis of the 11 studies involving 949 patients, including 393 deaths, yielded a pooled OR for death of 0.77 (95\% CI 0.58-1.03, p=0.079) (Fig. 2) with strong heterogeneity $\left(\mathrm{I}^{2}=70 \%, \mathrm{p}<0.001\right)$.

\section{Evaluation of publication bias}

In a funnel plot, the studies were marked almost linearly from the top of the pyramid to the left lower corner, with no studies in the right lower corner, which suggested strong publication bias. The Begg-Kendall test $(\tau=0.53, \mathrm{p}=0.024)$ also suggested the existence of significant publication bias (Fig. 3). Duval's trim and fill method estimated the presence of six potentially unreported cohorts. After these six potentially unpublished cohorts were filled, the pooled OR shifted to 1.11 (95\% CI 0.86-1.44, p=0.427) (Fig. 3, 4).

\section{Sensitivity analysis}

In addition to correcting the publication bias, we conducted sensitivity analyses by evaluating the degree of heterogeneity among the subgroups (Fig. 4). Consequently, the corticosteroid dose, study design, publication language, quality score and definition of ARDS were not found to be significant causes of heterogeneity.

Four subgroups showed significant heterogeneity. For example, the $I^{2}$ statistics for each subgroup according to the publication year, study weight, whether the patient was treated before or after day 14 and comorbidities were $81(\mathrm{p}=$ $0.023), 93(\mathrm{p}<0.001), 82(\mathrm{p}=0.018)$ and $82(\mathrm{p}<0.001)$, respectively.

\section{Discussion}

The current meta-analysis was unable to confirm the favorable impact of corticosteroid therapy on ARDS mortality. The analysis yielded a pooled OR for death of 0.77 (95\% CI $0.58-1.03, \mathrm{p}=0.079)$ with both strong heterogeneity $\left(\mathrm{I}^{2}=\right.$ $70 \%, \mathrm{p}<0.001)$ and publication bias $(\tau=0.52, \mathrm{p}=0.024)$ (Fig. 2). Heterogeneity refers to the degree of difference in results among included studies. Strong heterogeneity suggests that the validity of the meta-analysis is impaired, as the included studies reported inconsistent results.

Among the eight types of subgroups, the subgroups determined according to the study weight indicated the highest $\mathrm{I}^{2}$ statistics, with significant $\mathrm{p}$ values. While six studies with a weight of $<5 \%$ yielded an OR of 0.25 (95\% CI $0.13-0.48)$, the other five studies yielded an OR of 1.00 (95\% CI $0.73-$ 1.37). This difference, along with the results of the funnel 
Table. List of Meta-analyses and Included Original Studies.

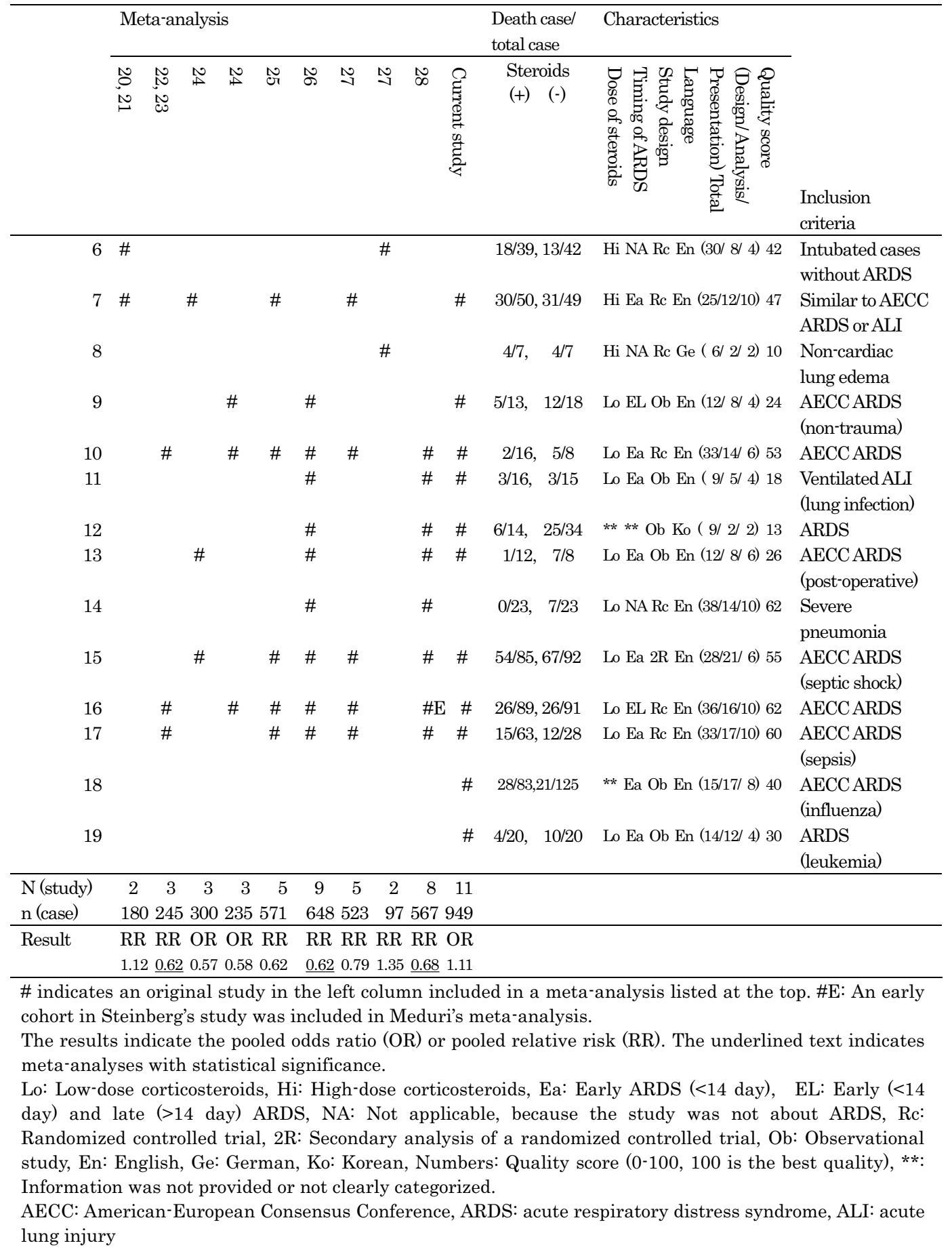

plot and Begg-Kendall test, suggests the existence of publication bias. Publication bias is caused by the tendency of editors and researchers to report positive results. Therefore, relatively small weighted studies showing favorable results are more likely to be published than small weighted studies showing harmful or null results. None of the previously published meta-analyses evaluated the degree of publication bias. The pooled values calculated for the 11 existing studies showed a marginally significant OR of 0.77 (95\% CI $0.58-1.03, \mathrm{p}=0.079$ ), as in previous meta-analyses. However, the OR increased to $\mathrm{OR}=1.11(95 \%$ CI $0.86-1.44, \mathrm{p}=0.427)$ after the publication bias was corrected. We would like to emphasize that existing articles often display publication bias, and no therapies should be rated based solely on the results of studies with a small sample size (Fig. 4).

The subgroups determined based on comorbidities were also associated with very strong heterogeneity. From the beginning, AECC-defined ARDS has included a variety of different disease entities $(1,2)$. Corticosteroid therapy may have a different therapeutic impact and side effects depending on the trigger event and existence of comorbidities. Both Varpula's and Brun-Buisson's studies included patients with 


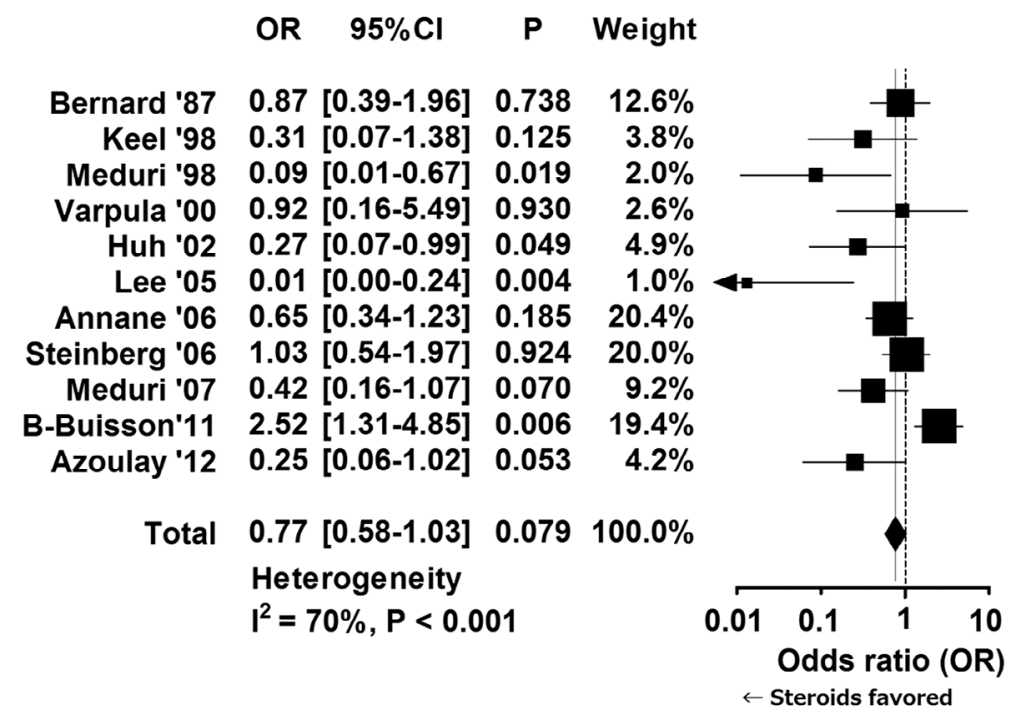

Figure 2. Forrest plot. The fixed-model meta-analysis of the 11 studies involving 949 patients, with 393 deaths, yielded a pooled OR for death of 0.77 (95\% CI 0.58-1.03, p=0.079), with strong heterogeneity $\left(I^{2}=70 \%, p<0.001\right)$. The error bars indicate $95 \%$ CIs. The vertical solid line indicates a pooled OR of 0.77. The vertical broken line indicates an OR of 1.0.
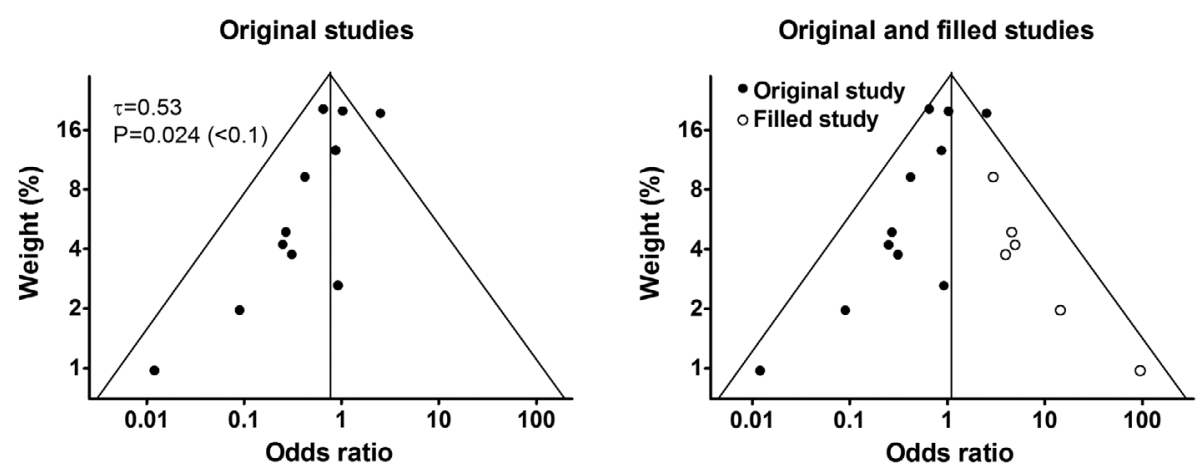

Figure 3. Funnel plots. Left: In the funnel plot, the studies were marked almost linearly from the top of the pyramid to the left lower corner, with no studies in the right lower corner, which suggested strong publication bias. The Begg-Kendall test $(\tau=0.53, p=0.024)$ also indicated the existence of significant publication bias. Right: Duval's trim and fill method estimated the presence of six potentially unreported cohorts. After these six potentially unpublished cohorts were filled, the pooled OR shifted to $1.11(95 \%$ CI $0.86-1.44, p=0.427)$.

pulmonary infection (Table). The pooled OR for these two cohorts was $2.24(95 \%$ CI 1.21-4.13, p=0.01), without heterogeneity $\left(I^{2}=7, p\right.$ for heterogeneity $\left.=0.30\right)$. Immunosuppression due to corticosteroid treatment may be associated with disadvantages in treating patients with pulmonary infections $(11,18,38)$. On the other hand, Azoulay's study suggested that corticosteroid treatment is favorable for patients with ARDS arising from leukemic pulmonary involvement as this treatment prevents the development of lysis pneumopathy. Patients with leukemic pulmonary involvement often satisfy the AECC criteria for ARDS, although the pathophysiology is different from that of diffuse alveolar damage (19). Further research is needed to determine pathophysiology- or trigger event-specific treatment strategies.

Articles published prior to 2005 reported significantly more favorable ORs, with considerable heterogeneity $\left(\mathrm{I}^{2}=82\right.$, $\mathrm{p}=0.018$ ), compared to those published since 2006. This finding may represent the history of research on the current topic. Various relatively recent studies with comparatively large numbers of patients were designed to confirm the favorable outcomes observed in earlier trials, although the relatively recent large-scale studies were unable to confirm such outcomes (Fig. 2, 4).

Corticosteroids mitigate inflammation in many ways, interacting with intracellular receptors that mediate the transcription of proinflammatory genes, particularly nuclear factor kappa beta (2). Given the key role of innate immune dysregulation in the pathogenesis of ARDS, researchers have conducted trials based on the belief that corticosteroid treatment can control ARDS progression $(6,7,10)$. On the contrary, it is well-known that side effects, such as immunosup- 


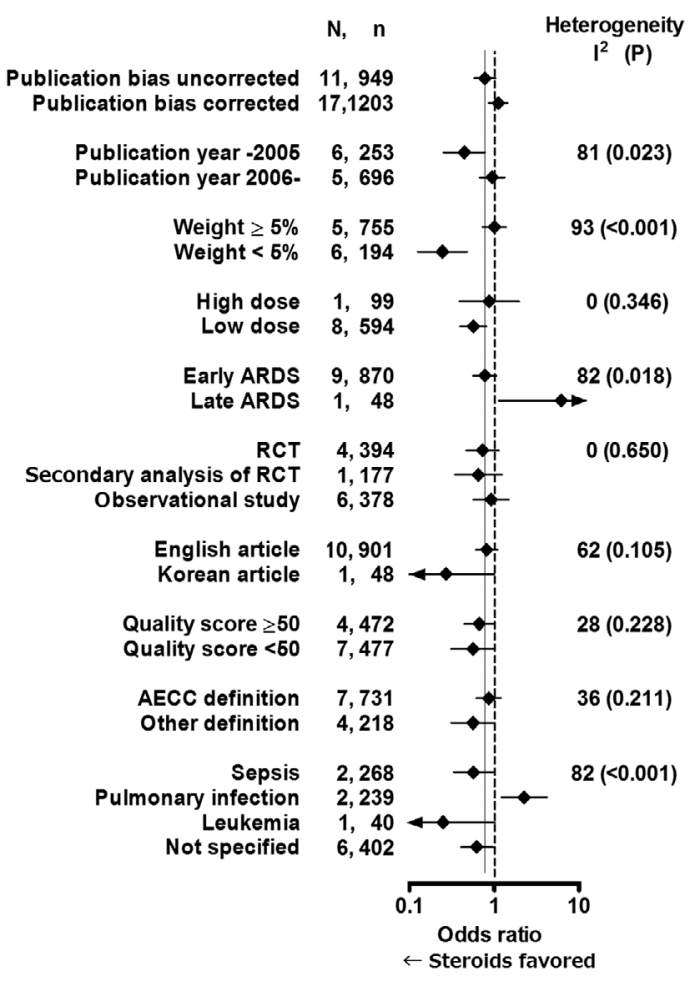

Figure 4. Sensitivity analyses. N: Number of studies, n: Number of cases. The error bars indicate $95 \%$ confidence intervals. The vertical solid line indicates a pooled odds ratio of 0.77 calculated for all studies, without publication bias correction. The broken line indicates an odds ratio of 1.0. In order to evaluate the pooled odds ratios for "early/late ARDS," Steinberg's cohort was divided into an early and late cohort.

pression and hyperglycemia, resulting from corticosteroid therapy have considerable disadvantages in patients in critical condition.

A limitation of the current analysis is that it included non-RCT studies, studies with low quality scores and studies written in languages other than English. A meta-analysis consisting of only high-quality RCTs would be attractive, although this not a possible strategy for the current topic, as the number of published RCTs was limited. In recent years, meta-analyses using non-RCT articles have become widely accepted, and the number of published meta-analyses including non-RCT studies has increased accordingly (39). Furthermore, the current sensitivity analysis did not detect heterogeneity among the subgroups divided according to the study design. We believe that the inclusion of observational studies did not result in considerable bias. Similarly, the sensitivity analyses suggested that the inclusion of nonEnglish articles, studies with low quality scores and studies using non-AECC definitions of ARDS did not have a significant effect on the results of the meta-analysis. Another limitation concerns the inclusion criteria. Currently, the most widely accepted definition of ARDS is based on the clinical diagnosis. However, clinically confirmed ARDS, i.e. ARDS diagnosed according to the AECC or Berlin definition, is apparently heterogeneous and not always accompanied by pathological findings of DAD $(5,29)$. This may explain why most of previous trials have failed to identify effective treatment options, with the exception of ventilation and supportive care. Although some treatments have been shown to have excellent therapeutic effects in limited subgroups, the benefits were diluted in the overall study population.

In conclusion, we were unable to confirm, based on the data of published studies, the favorable impact of corticosteroid therapy on the mortality of overall ARDS cases. In this study, the published articles had strong publication bias, and previous meta-analyses may have been affected by this publication bias. Further research focusing on pathophysiology- or trigger event-specific ARDS is anticipated.

The work was performed at: Department of Internal Medicine and Clinical Immunology, Yokohama City University Graduate School of Medicine

The authors state that they have no Conflict of Interest (COI).

\section{References}

1. Bernard GR, Artigas A, Brigham KL, et al. The AmericanEuropean Consensus Conference on ARDS. Definitions, mechanisms, relevant outcomes, and clinical trial coordination. Am J Respir Crit Care Med 149: 818-824, 1994.

2. Ware LB, Matthay MA. The acute respiratory distress syndrome. N Engl J Med 342: 1334-1349, 2000.

3. Ashbaugh DG, Bigelow DB, Petty TL, Levine BE. Acute respiratory distress in adults. Lancet 2: 319-323, 1967.

4. Petty TL, Ashbaugh DG. The adult respiratory distress syndrome. Clinical features, factors influencing prognosis and principles of management. Chest 60: 233-239, 1971.

5. Artigas A, Bernard GR, Carlet J, et al. The American-European Consensus Conference on ARDS, part 2: ventilatory, pharmacologic, supportive therapy, study design strategies, and issues related to recovery and remodeling. Acute respiratory distress syndrome. Am J Respir Crit Care Med 157: 1332-1347, 1998.

6. Weigelt JA, Norcross JF, Borman KR, Snyder WH 3rd. Early steroid therapy for respiratory failure. Arch Surg 120: 536-540, 1985.

7. Bernard GR, Luce JM, Sprung CL, et al. High-dose corticosteroids in patients with the adult respiratory distress syndrome. $\mathrm{N}$ Engl J Med 317: 1565-1570, 1987.

8. Laggner AN, Lenz K, Base W, et al. Effect of high-dose prednisolone on lung fluid in patients with non-cardiogenic lung edema. Wien Klin Wochenschr 99: 245-249, 1987 (in German, Abstract in English).

9. Keel JB, Hauser M, Stocker R, Baumann PC, Speich R. Established acute respiratory distress syndrome: benefit of corticosteroid rescue therapy. Respiration 65: 258-264, 1998.

10. Meduri GU, Headley AS, Golden E, et al. Effect of prolonged methylprednisolone therapy in unresolving acute respiratory distress syndrome: a randomized controlled trial. JAMA 280: 159165, 1998.

11. Varpula T, Pettilä V, Rintala E, Takkunen O, Valtonen V. Late steroid therapy in primary acute lung injury. Intensive Care Med 26: 526-531, 2000.

12. Huh JW, Lim CM, Jegal YJ, et al. The effect of steroid therapy in patients with late ARDS. Tuberc Respir Dis 52: 376-384, 2002 (in Korean, Abstract in English).

13. Lee HS, Lee JM, Kim MS, Kim HY, Hwangbo B, Zo JI. Lowdose steroid therapy at an early phase of postoperative acute respi- 
ratory distress syndrome. Ann Thorac Surg 79: 405-410, 2005.

14. Confalonieri M, Urbino R, Potena A, et al. Hydrocortisone infusion for severe community-acquired pneumonia: a preliminary randomized study. Am J Respir Crit Care Med 171: 242-248, 2005.

15. Annane D, Sébille V, Bellissant E; Ger-Inf-05 Study Group. Effect of low doses of corticosteroids in septic shock patients with or without early acute respiratory distress syndrome. Crit Care Med 34: 22-30, 2006.

16. Steinberg KP, Hudson LD, Goodman RB, et al. Efficacy and safety of corticosteroids for persistent acute respiratory distress syndrome. N Engl J Med 354: 1671-1684, 2006.

17. Meduri GU, Golden E, Freire AX, et al. Methylprednisolone infusion in early severe ARDS: results of a randomized controlled trial. Chest 131: 954-963, 2007.

18. Brun-Buisson C, Richard JC, Mercat A, Thiébaut AC, Brochard L; REVA-SRLF A/H1N1v 2009 Registry Group. Early corticosteroids in severe influenza $\mathrm{A} / \mathrm{H} 1 \mathrm{~N} 1$ pneumonia and acute respiratory distress syndrome. Am J Respir Crit Care Med 183: 1200-1206, 2011.

19. Azoulay É, Canet E, Raffoux E, et al. Dexamethasone in patients with acute lung injury from acute monocytic leukaemia. Eur Respir J 39: 648-653, 2012.

20. Adhikari N, Burns KE, Meade MO. Pharmacologic therapies for adults with acute lung injury and acute respiratory distress syndrome. Cochrane Database Syst Rev 4: CD004477, 2004.

21. Adhikari N, Burns KE, Meade MO. Pharmacologic treatments for acute respiratory distress syndrome and acute lung injury: systematic review and meta-analysis. Treat Respir Med 3: 307-328, 2004.

22. Meduri GU. There is no illumination in speculation: additional data in support of methylprednisolone treatment in ARDS. Chest 132: 1097-1100, 2007

23. Meduri GU, Marik PE, Chrousos GP, et al. Steroid treatment in ARDS: a critical appraisal of the ARDS network trial and the recent literature. Intensive Care Med 34: 61-69, 2008.

24. Agarwal R, Nath A, Aggarwal AN, Gupta D. Do glucocorticoids decrease mortality in acute respiratory distress syndrome? A metaanalysis. Respirology 12: 585-590, 2007.

25. Peter JV, John P, Graham PL, Moran JL, George IA, Bersten A. Corticosteroids in the prevention and treatment of acute respiratory distress syndrome (ARDS) in adults: meta-analysis. BMJ 336: 1006-1009, 2008.

26. Tang BM, Craig JC, Eslick GD, Seppelt I, McLean AS. Use of corticosteroids in acute lung injury and acute respiratory distress syndrome: a systematic review and meta-analysis. Crit Care Med 37: 1594-1603, 2009.

27. Lamontagne F, Briel M, Guyatt GH, Cook DJ, Bhatnagar N, Meade M. Corticosteroid therapy for acute lung injury, acute respiratory distress syndrome, and severe pneumonia: a meta-analysis of randomized controlled trials. J Crit Care 25: 420-435, 2010.

28. Meduri GU, Rocco PR, Annane D, Sinclair SE. Prolonged glucocorticoid treatment and secondary prevention in acute respiratory distress syndrome. Expert Rev Respir Med 4: 201-210, 2010.

29. ARDS Definition Task Force. Acute respiratory distress syndrome: the Berlin Definition. JAMA 307: 2526-2533, 2012.

30. Liberati A, Altman DG, Tetzlaff J, et al. The PRISMA statement for reporting systematic reviews and meta-analyses of studies that evaluate healthcare interventions: explanation and elaboration. BMJ 339: b2700, 2009.

31. Chalmers TC, Smith H Jr, Blackburn B, et al. A method for assessing the quality of a randomized control trial. Control Clin Trials 2: 31-49, 1981.

32. Greenland S. Quantitative methods in the review of epidemiologic literature. Epidemiol Rev 9: 1-30, 1987.

33. Higgins JP, Thompson SG, Deeks JJ, Altman DG. Measuring inconsistency in meta-analyses. BMJ 327: 557-560, 2003.

34. Begg CB, Mazumdar M. Operating characteristics of a rank correlation test for publication bias. Biometrics 50: 1088-1101, 1994.

35. Duval S, Tweedie R. Trim and fill: a simple funnel-plot-based method of testing and adjusting for publication bias in metaanalysis. Biometrics 56: 455-463, 2000.

36. Sutton AJ, Duval SJ, Tweedie RL, Abrams KR, Jones DR. Empirical assessment of effect of publication bias on meta-analyses. BMJ 320: 1574-1577, 2000.

37. Altman DG, Bland JM. Interaction revisited: the difference between two estimates. BMJ 326: 219, 2003.

38. Snijders D, Daniels JM, de Graaff CS, van der Werf TS, Boersma WG. Efficacy of corticosteroids in community-acquired pneumonia: a randomized double-blinded clinical trial. Am J Respir Crit Care Med 18: 975-982, 2010.

39. Stroup DF, Berlin JA, Morton SC, et al. Meta-analysis of observational studies in epidemiology: a proposal for reporting. Metaanalysis Of Observational Studies in Epidemiology (MOOSE) group. JAMA 283: 2008-2012, 2000.

(C) 2015 The Japanese Society of Internal Medicine http://www.naika.or.jp/imonline/index.html 